Department: Visualization Viewpoints

Editor: Theresa-Marie Rhyne, theresamarierhyne@gmail.com

\title{
Reaching Broader Audiences with Data Visualization
}

\author{
Bongshin Lee \\ Microsoft Research
}

Eun Kyoung Choe

University of Maryland, College Park

Petra Isenberg

Inria

Kim Marriott

Monash University

John Stasko

Georgia Institute of Technology

\begin{abstract}
The visualization research community can and should reach broader audiences beyond data-savvy groups of people, because these audiences could also greatly benefit from visual access to data. In this paper, we discuss four research topics-personal data visualization, data visualization on mobile devices, inclusive data visualization, and multimodal interaction for data visualization-that, individually and collaboratively, would help us reach broader audiences with data visualization, making data more accessible.
\end{abstract}

VISUALIZATION research has become a burgeoning field encompassing many established and emerging sub areas, such as biological data visualization, visualization for cyber security, and visual analytics for healthcare, among others. Such research progression has empowered experts dealing with massive data (e.g., researchers, biologists, data analysts) to apply and leverage visualizations in their work. However, current visualization research often leaves out broader audiences, who could also benefit from innovations in visualization research. Compared to the experts mentioned above, these wider audiences have fundamental differences in their needs, motivations, and goals as well as in their capabilities. We as a visualization research community should attune to these broader audiences as we see great opportunities to make practical impacts in their everyday life leveraging data visualization.

In this paper, we discuss four research topics that can help us reach broader audiences with data visualization, making data more accessible. Ever growing capabilities of personal data collections provide a great opportunity for people 
to make positive changes in their lives through better understanding of themselves [1]. The need and use of data visualization on mobile devices (e.g., tablets, smartphones, smartwatches) is increasingly prevalent in practice, enabling people to access data without relying on a desktop or laptop [2]. Advancements in both software and hardware technologies give us opportunities to provide people with disabilities with greater access to data visualization. Multimodal interaction offers many potential benefits for data visualization [3], where conventional input modalities such as keyboard and mouse are not available (e.g., on mobile devices) or when users have limited capabilities (e.g., people with low visualization literacy, blind people).

We do not claim that these four topics are comprehensive or more important than others in reaching broader audiences. However, we posit that they each can enrich the value of visualization for broader audiences, and more importantly they together can generate synergy to enhance the capabilities of the general public to better access and utilize data. They also suggest a wealth of important and interesting research questions for the visualization community.

We first describe the characteristics of these broader audiences. We then discuss why each of our four topics deserves more efforts from the visualization community, along with some ongoing (or stagnant) efforts and recent projects.

\section{CHARACTERIZING BROADER AUDIENCES}

Going beyond data-savvy people such as scientists and researchers that traditional visualization research has mainly targeted, our potential audiences include people who have the following characteristics. First, they have a wide range of data and visualization literacy. While some data enthusiasts are familiar with more sophisticated charts (e.g., scatterplots, node-link diagrams, treemaps), others may only have a basic knowledge of simple charts (e.g., bar charts, line charts, pie charts). Lack of prior experience with accessible charts is a particular issue for people who are blind.

Our broader audiences are likely to have different usage contexts and goals. They may not care about gaining insights from a detailed data exploration but use data visualization for more casual purposes [4] such as for fun and curiosity, as well as for simple data access. On the other hand, some people who are not formally trained in data analysis or statistics may still need to use data visualizations to fulfill their tasks.

As these broader audiences expand the usage contexts, data access environments will vary as well, going beyond the stationary desktop environment. People will use data visualization on the go or while lying down on a sofa, both likely using mobile devices.

These novel characteristics and contexts pose unique challenges and immense opportunities for visualization researchers, which we discuss in the following sections.

\section{PERSONAL DATA VISUALIZATION}

People nowadays have many opportunities to collect and interact with data due to easy access to low-cost sensors, mobile devices, and applications. From the data collected with these devices and applications, people can learn and understand various aspects of their lives-such as health, social connectedness, and productivity. Enhanced awareness of one's own behaviors can be critical in setting and achieving diverse personal goals.

In the visualization and human-computer interaction research communities, researchers have been working to help people better collect data and identify personal insights. Called Personal Informatics, this growing field of research deals with supporting ways in which we can leverage personal data, by understanding people's tracking needs and usage, and by designing, developing, and evaluating new technologies. This field intersects with the visualization community in the goal of making it easy for people to better understand their data and communicate personal insights. In 2014, Huang et al. published a survey paper on personal visualization and personal visual analytics, mapping the personal informatics work through the lens of visualization research [1].

At VIS 2015, researchers interested in this topic held a workshop, "Personal Visualization: Exploring Data in Everyday Life," (www.vis4me.com/personalvis15) with a long term goal of empowering people to make improvements to their lives and communities through advances in personal visualization. Fol- 

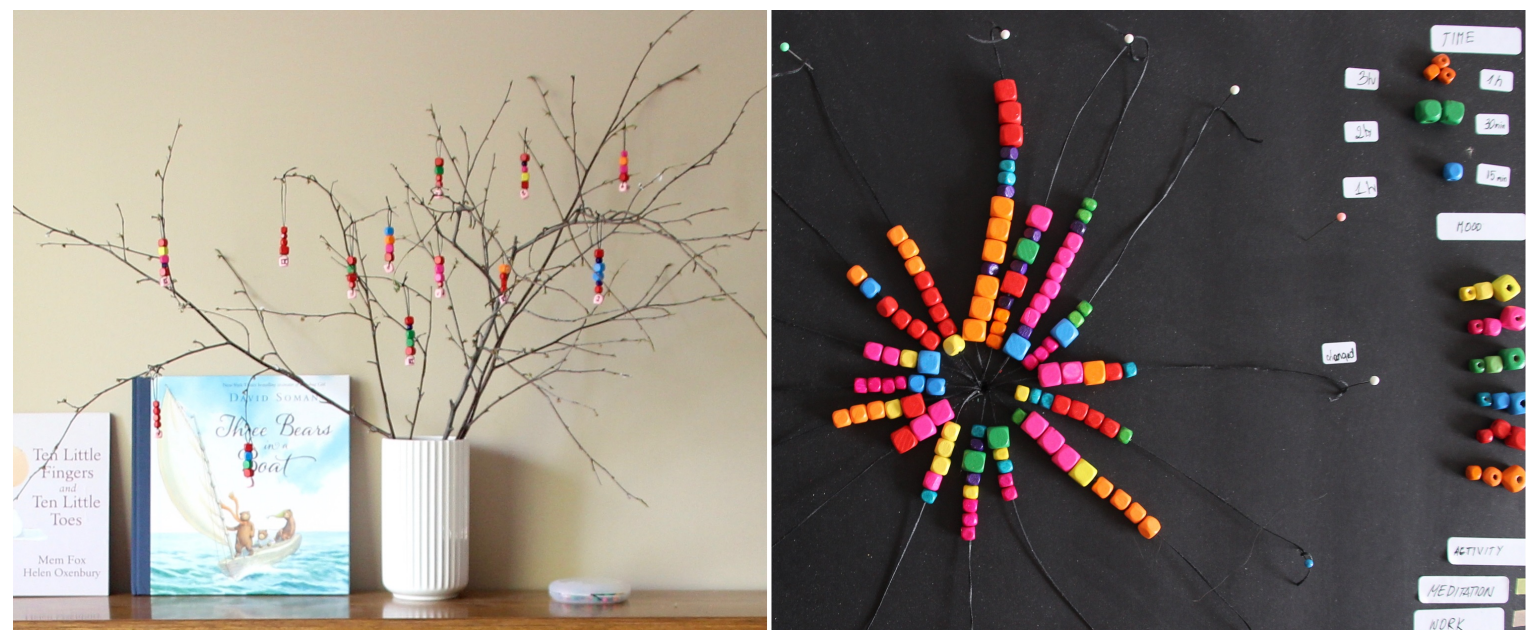

Figure 1. Examples of data physicalization with personal data [5] (Courtesy of Alice Thudt). (Left) A bead ornament daily to decorate branches with 4-hour interval represented by one bead with size showing her mood and colour showing whether she was active, social, or home. (Right) a participant's six different activities (e.g., meditation, work) with different coloured beads, each representing one hour, with their size showing enjoyment.

lowing this workshop, however, only a limited number of researchers have continued to work at the intersection of visualization and personal informatics (e.g., data physicalization shown in Figure 1 [5], research methods in personal visualization [6]). We call for further efforts and contributions to empower the general public while addressing meaningful visualization challenges.

To advance and reach the full potential of personal data visualization, we need to explore ways to improve the general public's visualization literacy. Although visualizations are heavily used even in the elementary school curricula, this does not necessarily mean that basic visualization skills are being taught [7]: Chevalier et al. found that teachers perceive visualizations as "intuitive" and therefore do not actively teach them. Visualization researchers should continue to design and develop novel visualization techniques for the general public. However, such efforts would be meaningful only if a certain level of visualization literacy is assured.

Going beyond improving basic visualization literacy for the general public, there are cases where people can benefit from being able to explore data. For example, dedicated self-trackers (also known as Quantified Selfers) commonly collect and explore data, and share interesting insights drawn from their data [8]. However, a majority of people find it challenging to construct appropriate visualizations, which involves translating questions into data attributes and choosing visual mappings [9]. Such skills should be more easily attainable for people who want to go a step further in exploring their dataeither through teaching existing tools such as Tableau (www.tableau.com) and Microsoft Power BI (www.powerbi.com) or innovating new tools that can make the visualization construction and exploration process much easier to learn and use.

To design effective personal visualization, designers and researchers should better understand the personal data context to inform the visualization design. These contexts are often diverse, onthe-go, situated, and personalized. Creative ways and methods, such as leveraging wearable cameras to capture a variety of in-situ contexts [10], are being developed by and introduced to the research community. Visualization researchers may leverage a variety of in-situ study methods both for formative and evaluative purposes that would suit personal data contexts. We encourage visualization researchers to proactively collaborate with the broader HCI communities to infuse and discuss design and UX aspects.

\section{DATA VISUALIZATION ON MOBILE DEVICES}

Smartphone ownership has surpassed personal computer (i.e., PC and laptop) ownership 
(tinyurl.com/qnhbf7b). Smartphones are being used for a broad range of activities where visualization can help to provide easier access to data. This involves data delivered from specific apps on smartphones and tablets related to personally relevant data such as weather, personal finances, fitness tracking, and health monitoring, as well as to data related to business- and work-related information including calendars, measurements taken, and products sold. Large journalism outlets have been producing data-driven stories with their mobile readership in mind or even using a mobilefirst design concept. In addition, many mobile apps include simple data visualizations with additional goals to attract audiences-fitness tracking apps are a prime example (Figure 2).

The accumulated knowledge in the visualization community, however, only helps the designers of mobile apps and responsive websites to a limited extent. As visualization research in the past focused on desktop environments, it is often unclear if guidelines will hold for the fundamentally different mobile environments with changed physical display sizes, interaction mechanisms, target audiences, and usage contexts. For example, we need to rethink the traditional WIMP (windows, icons, menus, pointer) interface via direct manipulation for mobile devices. Mobile visualizations cannot rely on precise selections or large cascading menus for data- and visualizationrelated operations. In addition, common interaction modes such as keyboard shortcuts to select multiple data points, right-clicks to choose a desired operation, and mouse-over to show details on demand become unavailable. Thus, mobile visualization requires new approaches to interaction design and potentially needs to be operable with a reduced number of manipulations for making changes to data, representation, presentation, and view parameters.

The difficulties of designing visualizations for mobile, however, are not only related to the interaction concept. Mobile devices these days often have very high-resolution displays but the limited physical size requires rethinking minimal sizes for the display of data points and reference structures (e.g., grids, labels, tickmarks). Scalability regarding smaller rather than larger display sizes becomes a problem for mobile visualizations and we need more perceptual studies to understand

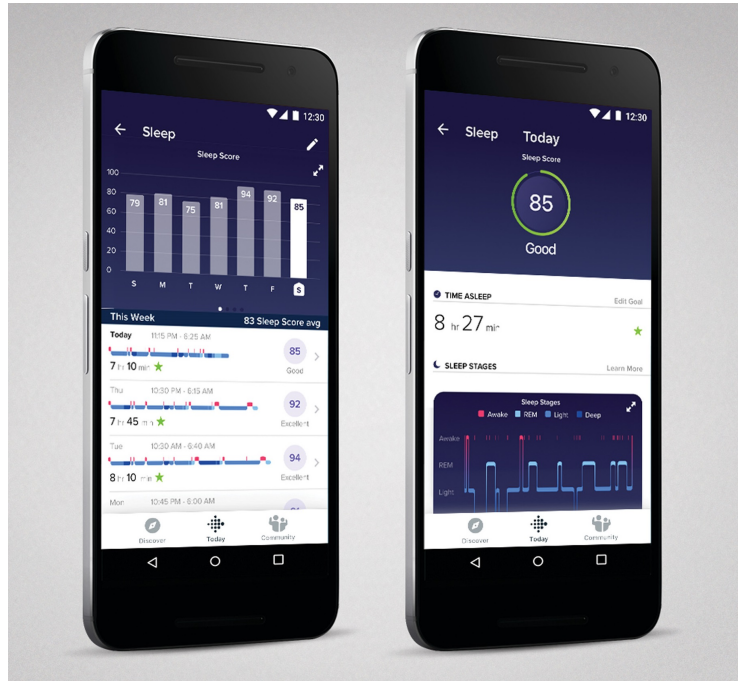

Figure 2. The Fitbit mobile app shows daily sleep scores with a standard bar chart (left) while displaying different sleep stages with a custom chart (right).

the effects of minimizing different types of visualizations and their reference structures.

A further design challenge for mobile devices arises from varying and unconventional usage contexts compared to desktop computer use. As in the case of personal data contexts, mobile devices are used on the go, with moving observers and under varying lighting conditions. Quick information access-at a glance-becomes a new usage context for which there is little concrete design advice. Although there has been some research on ambient or peripheral displays in the broader HCI community, it would be important to explore if and how they transfer to the visualization context.

Recently, there have been two efforts in establishing a community around mobile data visualization: (1) the "Data Visualization on Mobile Devices" workshop at CHI 2018 [2] (mobilevis.github.io) and (2) the "Mobile Data Visualization" Dagstuhl Seminar in July 2019 [11] (tinyurl.com/mobilevis-dagstuhl). We call for visualization researchers to attract researchers from related communities to advance this important and exciting topic.

\section{INCLUSIVE DATA VISUALIZATION}

Approximately 36 million people in the world are blind and an additional 217 million have moderate to severe vision impairment. This large cohort is currently excluded from the benefits of 


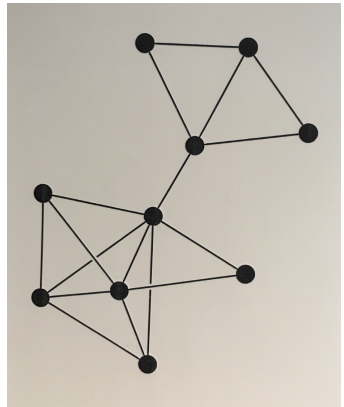

(a)

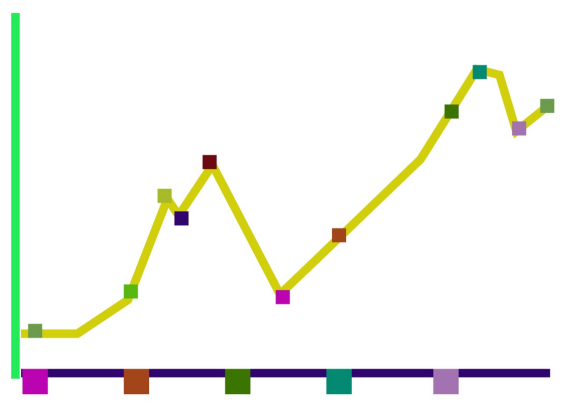

(b)

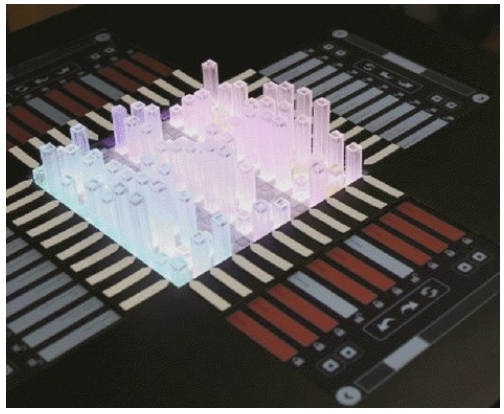

(c)

Figure 3. Possible ways of presenting data to blind users. (a) Traditionally spatial data is presented using tactile graphics. (b) A recent approach is to present data on a touchscreen with audio and sometimes haptic feedback. (c) A more futuristic possibility is to use dynamic physical displays [12] (Courtesy of Jason Alexander).

data visualization. While blindness or low-vision (BLV) is the most obvious kind of disability affecting access to data visualization, people with intellectual and developmental disabilities (IDDs) may also find it difficult to use existing visualization tools because of impaired cognitive processing [13], and people with physical disabilities may find it difficult to interact with them.

As the importance of data visualization increases and it is more widely used for datadriven reasoning and communication, this raises a serious equity issue, one that the data visualization community has almost totally ignored (one exception is color blindness). Furthermore, improving accessibility is not just of benefit to people with disabilities, but to all people. Flexible voice-activated solutions, for example, could allow people to use applications in a hands-free mode while cooking, crafting, or doing sports.

One consideration when designing more inclusive data visualization applications is the choice of interaction modalities. Clearly the more flexibility in input modality, the more accessible the application will be. As an indication of the challenge think about someone who does not have use of their limbs. How can they control a data visualization application? Is it possible to provide a natural language interface with eyegaze controlled object and region selection?

An even more fundamental rethink of data visualization is required if we wish to provide access to people who are blind. Data must now be communicated through sound or touch, rather than vision (see Figure 3). The most common way to present graphical information to someone who is blind is by providing an audio or braille description. However, while this can provide a high-level overview it necessarily loses detailed information and does not allow the reader to develop their own independent interpretation.

Accessibility guidelines recommend the use of raised line drawings, called tactile graphics, for graphics in which spatial relationships are important, such as maps, charts, diagrams, and graphs. Studies have shown that tactile graphics provide touch readers with many of the benefits that visualizations provide sighted readers. For example, a recent study compared a tactile nodelink diagram with a braille list representation of a network [14]. The blind participants found the node-link diagram intuitive and were faster and made less errors with this representation when counting the number of clusters or finding a path between two nodes.

Unfortunately, traditional tactile graphics are not well-suited to interactive exploration of data as each tactile graphic costs around US \$1 to print. Data exploration requires a refreshable display. While refreshable braille displays using peizo-electric actuated pins have been available for many years, currently larger displays suitable for displaying graphics using this technology are prohibitively expensive. Fortunately, low-cost refreshable braille displays using other approaches are about to become available, potentially allowing their use for interactive data visualization.

Data physicalization is a promising way of creating more accessible visualizations. The visualization community has started to explore physical data representations as a way of creating 
more engaging presentations (e.g., Figure 1), and the accessibility community is gaining considerable interest in the use of $3 \mathrm{D}$ printing to create accessible models for use in education and orientation and mobility training for BLV people. Commodity $3 \mathrm{D}$ printing now allows $3 \mathrm{D}$ models to be produced at the same cost as tactile graphics. However, standard 3D models have the same disadvantage as tactile graphics: they are static. Recently there has been research into dynamic data physicalization (e.g., Figure 3c [12]) but more research is required for these devices to become practical.

Inclusive data visualization raises a large number of research issues. These range from the design of new display technologies such as lowcost dynamic physical displays and new interfaces allowing hands-free operation, to the development of evidence based guidelines for the design of visualizations that are suitable for low vision. We particularly note the lack of research investigating intellectual and developmental disabilities (IDDs) and data visualization [13]. Research is required to investigate the impact of IDDs on understanding and how to design visualizations that are more accessible to this community. In general, making data visualization more accessible to people with various disabilities will require visualization researchers to partner with the accessibility community to ensure that the solutions developed really do meet the needs of the intended users.

\section{MULTIMODAL INTERACTION FOR DATA VISUALIZATION}

The majority of visualization applications developed to date, particularly those designed for analysts and experts, have provided a single method of interaction, namely direct manipulation via a mouse and keyboard. A multimodal interface, conversely, utilizes multiple modes of input such as touch, pen, speech, natural language, and gesture to allow a person to interact with and control the system.

To investigate the potential benefits of leveraging multiple input modalities, several visualization systems have combined at least two modes of input. For example, SketchInsight allows people to visually explore their data by interactively creating and manipulating charts with pen and touch interaction [16]. More recently, Orko (Fig- ure 4) combines speech-based natural language with touch-based direct manipulation to facilitate network-based data exploration [15].

These alternative methods of input, sometimes characterized as Post-WIMP [17], can help visualization reach the new audiences we have identified in the earlier section. Multimodal interaction helps us tackle the fundamental challenges in interaction design when a mouse and keyboard are not available, and when the display device is smaller and/or movable. This includes the case where a visualization is presented on a mobile device, as discussed earlier. Speech commands can replace operations typically entered through a keyboard or manipulated through a mouse, and they allow the removal of control panels that take up valuable display real estate. For example, speech interaction can enable easy data entry (e.g., date, time, date ranges), which is often tedious to do on mobile devices [18]. Such interfaces could allow people to easily explore time-oriented data (e.g., self-tracking data) on mobile devices.

We envision other scenarios where multimodal interaction could help bring visualization to novices. Natural language interaction, especially when combined with direct manipulation, can lower the barrier to access data by making it easy to express data-oriented questions without learning native database query languages or specific user interfaces [19]. Such interfaces could allow people to express questions about data more naturally, as they would in everyday conversation.

Multimodal interaction can also be used to make data visualization more accessible to people with disabilities. For example, one approach to support interactive exploration or consumption of data by BLV people is to present visualization on a touchscreen device using a mix of touch triggered audio and haptic feedback where the audio feedback includes both sonification and audio description (see Figure 3b). This approach has been explored for accessible graphing calculators (e.g., [20]), but to the best of our knowledge has not been employed and evaluated more broadly for interactive exploration or consumption of data. Speech input also harbors great potential to assist people whose motor disabilities make the use of traditional input devices such as a mouse, keyboard, and pen difficult or impossible. 


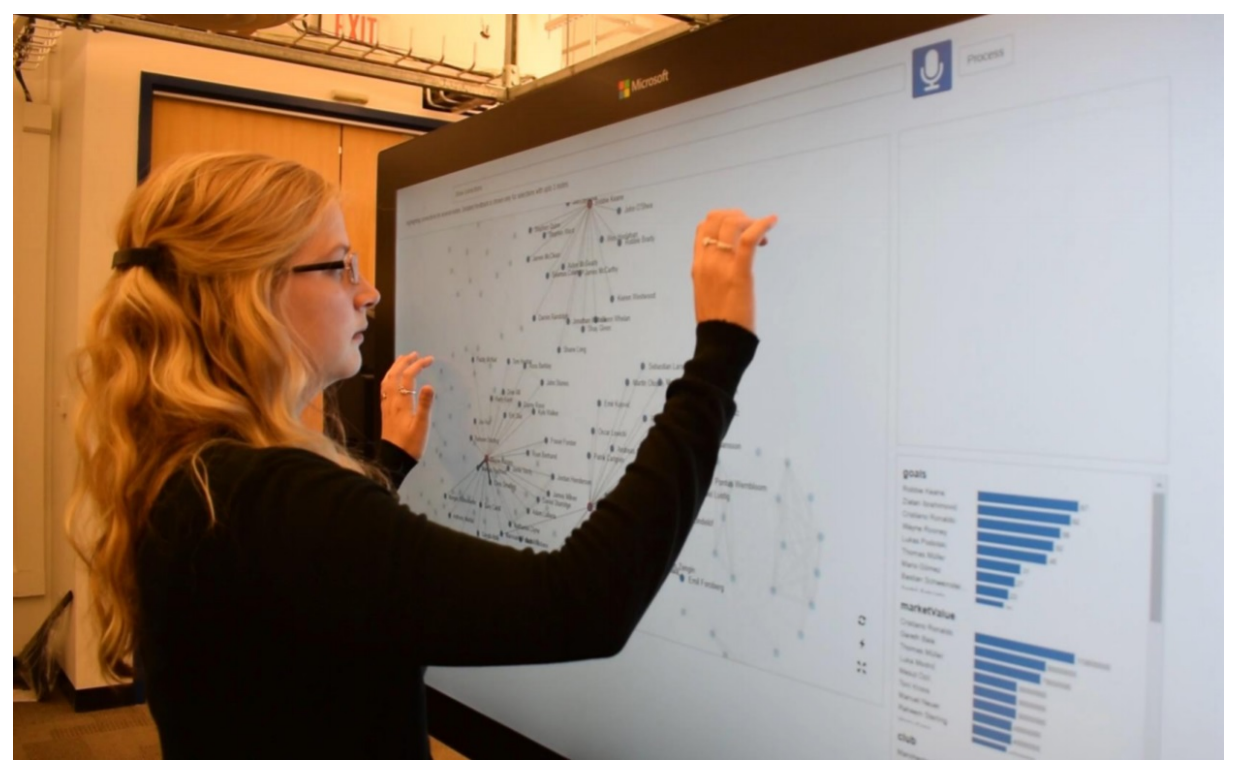

Figure 4. Orko is a network visualization tool that facilitates multimodal interaction by combining natural language (with speech) and direct manipulation (with touch) [15].

The "Multimodal Interaction for Data Visualization" workshop at AVI 2018 [3] (multimodalvis.github.io) is the initial attempt to build a community of multimodal visualization researchers and establish an agenda for research on multimodal interactions for and with visualization. We call for follow-up efforts from the visualization research community to advance this topic, investigating important and interesting research questions.

\section{OTHER RESEARCH TOPICS}

We acknowledge that other topics beyond these four can help visualization reach broader audiences. Some of them are covered by other viewpoints articles or mentioned earlier in this article. For example, visualization literacy [7] is an important topic. It is critical underpinning for personal data visualization and also plays a role in increasing accessibility. Data-driven storytelling [21] has the potential to improve communication of information to targeted audiences. Data physicalization can encourage affective, physical, intellectual, and social engagement for various target groups [22]. In addition, research into visualization for and with AI, could help to understand and target the needs of wider user groups. We hope to see other interesting topics emerge, of course, also around topics outside the ones we focused on.

\section{CONCLUSION}

The field of information visualization has made great strides, empowering experts to deal with massive data leveraging visualization in their work. However, we note that visualization research falls short on serving a larger group of people who can also benefit from data visualization. As they have a wider range of data and visualization literacy as well as more diverse usage contexts and goals, these broader audiences pose interesting challenges and opportunities for visualization researchers. We specifically called for four research topics-personal data visualization, data visualization on mobile devices, inclusive data visualization, and multimodal interaction for data visualization: they would help us reach broader audiences and increase the practical impact of data visualization.

\section{REFERENCES}

1. D. Huang, M. Tory, B. A. Aseniero, L. Bartram, S. Bateman, S. Carpendale, A. Tang, and R. Woodbury, "Personal visualization and personal visual analytics," IEEE Transactions on Visualization and Computer Graphics, vol. 21, no. 3, pp. 420-433, 2014.

2. B. Lee, M. Brehmer, P. Isenberg, E. K. Choe, R. Langner, and R. Dachselt, "Data visualization on 
mobile devices," in Extended Abstracts of the $2018 \mathrm{CHI}$ Conference on Human Factors in Computing Systems. ACM, 2018, p. W07.

3. B. Lee, A. Srinivasan, J. Stasko, M. Tory, and V. Setlur, "Multimodal interaction for data visualization," in Proceedings of the 2018 International Conference on Advanced Visual Interfaces. ACM, 2018, p. 11.

4. Z. Pousman, J. Stasko, and M. Mateas, "Casual information visualization: Depictions of data in everyday life," IEEE Transactions on Visualization and Computer Graphics, vol. 13, no. 6, pp. 1145-1152, Nov. 2007.

5. A. Thudt, U. Hinrichs, S. Huron, and S. Carpendale, "Self-reflection and personal physicalization construction," in Proceedings of the $2018 \mathrm{CHI}$ Conference on Human Factors in Computing Systems. ACM, 2018, p. 154.

6. A. Thudt, B. Lee, E. K. Choe, and S. Carpendale, "Expanding research methods for a realistic understanding of personal visualization," IEEE computer graphics and applications, vol. 37, no. 2, pp. 12-18, 2017.

7. F. Chevalier, N. Henry Riche, B. Alper, C. Plaisant, J. Boy, and N. Elmqvist, "Observations and reflections on visualization literacy in elementary school," IEEE computer graphics and applications, vol. 38, no. 3, pp. 21-29, 2018.

8. E. K. Choe, B. Lee, and m. c. schraefel, "Characterizing visualization insights from quantified selfers' personal data presentations," IEEE computer graphics and applications, vol. 35, no. 4, pp. 28-37, 2015.

9. L. Grammel, M. Tory, and M.-A. Storey, "How information visualization novices construct visualizations," IEEE Transactions on Visualization and Computer Graphics, vol. 16, no. 6, pp. 943-952, 2010.

10. S. Pizza, B. Brown, D. McMillan, and A. Lampinen, "Smartwatch in vivo," in Proceedings of the $2016 \mathrm{CHI}$ Conference on Human Factors in Computing Systems. ACM, 2016, pp. 5456-5469.

11. E. K. Choe, R. Dachselt, P. Isenberg, and B. Lee, "Mobile data visualization (dagstuhl seminar 19292)." Schloss Dagstuhl-Leibniz-Zentrum fuer Informatik, 2019.

12. F. Taher, Y. Jansen, J. Woodruff, J. Hardy, K. Hornbæk, and J. Alexander, "Investigating the use of a dynamic physical bar chart for data exploration and presentation," IEEE Transactions on Visualization and Computer Graphics, vol. 23, no. 1, pp. 451-460, 2016.

13. K. Wu, S. Tanis, and D. Szafir, "Designing communicative visualization for people with intellectual developmental disabilities," in Workshop on Visualization for Communication (VisComm). OSF Preprints, 2019.
14. Y. Yang, K. Marriott, M. Butler, C. Goncu, and L. M. Holloway, "Tactile presentation of network data: Text, matrix or diagram?" in Proceedings of the $2020 \mathrm{CHI}$ Conference on Human Factors in Computing Systems. ACM, 2020, in press.

15. A. Srinivasan and J. Stasko, "Orko: Facilitating multimodal interaction for visual exploration and analysis of networks," IEEE Transactions on Visualization and Computer Graphics, vol. 24, no. 1, pp. 511-521, 2018.

16. B. Lee, G. Smith, N. Henry Riche, A. Karlson, and S. Carpendale, "Sketchinsight: Natural data exploration on interactive whiteboards leveraging pen and touch interaction," in 2015 IEEE Pacific Visualization Symposium (PacificVis). IEEE, 2015, pp. 199-206.

17. B. Lee, P. Isenberg, N. Henry Riche, and S. Carpendale, "Beyond mouse and keyboard: Expanding design considerations for information visualization interactions," IEEE Transactions on Visualization and Computer Graphics, vol. 18, no. 12, pp. 2689-2698, 2012.

18. E. K. Choe, B. Lee, and S.-W. Hwang, "Personal data exploration with speech on mobile devices," in AVI 2018 Workshop on Multimodal Interactoin for Data Visualization, 2018.

19. V. Setlur, S. E. Battersby, M. Tory, R. Gossweiler, and A. X. Chang, "Eviza: A natural language interface for visual analysis," in Proceedings of ACM UIST '16, 2016, pp. 365-377.

20. C. Goncu and K. Marriott, "Gracalc: An accessible graphing calculator," in Proceedings of the 17th International ACM SIGACCESS Conference on Computers \& Accessibility. ACM, 2015, pp. 311-312.

21. B. Lee, N. H. Riche, P. Isenberg, and S. Carpendale, "More than telling a story: Transforming data into visually shared stories," IEEE computer graphics and applications, vol. 35, no. 5, pp. 84-90, 2015.

22. Y. Wang, A. Segal, R. Klatzky, D. F. Keefe, P. Isenberg, J. Hurtienne, E. Hornecker, T. Dwyer, and S. Barrass, "An emotional response to the value of visualization," IEEE computer graphics and applications, vol. 39, no. 5, pp. 8-17, 2019.

Bongshin Lee is a Sr. Principal Researcher at Microsoft Research. Contact her at bongshin@microsoft.com.

Eun Kyoung Choe is an Assistant Professor in the College of Information Studies at the University of Maryland, College Park. Contact her at choe@umd.edu.

Petra Isenberg is a Research Scientist in visualiza- 
tion with Inria. Contact her at petra.isenberg@inria.fr.

Kim Marriott is a Professor at Monash University. Contact him at kim.marriott@monash.edu.

John Stasko is a Regents Professor in the School of Interactive Computing and the Director of the Information Interfaces Research Group at the Georgia Institute of Technology. Contact him at stasko@cc.gatech.edu. 